\title{
On Fractional Order Hybrid Differential Equations
}

\author{
Mohamed A. E. Herzallah ${ }^{1,2}$ and Dumitru Baleanu ${ }^{3,4,5}$ \\ ${ }^{1}$ Faculty of Science, Zagazig University, Zagazig, Egypt \\ ${ }^{2}$ College of Science in Zulfi, Majmaah University, Saudi Arabia \\ ${ }^{3}$ Department of Chemical and Materials Engineering, Faculty of Engineering, King Abdulaziz University, \\ Jeddah, Saudi Arabia \\ ${ }^{4}$ Department of Mathematics Computer Science, Cankaya University, 06530 Ankara, Turkey \\ ${ }^{5}$ Institute of Space Sciences, P.O. BOX, MG-23, 76900 Magurele-Bucharest, Romania
}

Correspondence should be addressed to Mohamed A. E. Herzallah; m_herzallah75@hotmail.com

Received 2 December 2013; Accepted 20 February 2014; Published 25 March 2014

Academic Editor: Robert A. Van Gorder

Copyright (c) 2014 M. A. E. Herzallah and D. Baleanu. This is an open access article distributed under the Creative Commons Attribution License, which permits unrestricted use, distribution, and reproduction in any medium, provided the original work is properly cited.

We develop the theory of fractional hybrid differential equations with linear and nonlinear perturbations involving the Caputo fractional derivative of order $0<\alpha<1$. Using some fixed point theorems we prove the existence of mild solutions for two types of hybrid equations. Examples are given to illustrate the obtained results.

\section{Introduction}

Fractional calculus is a field of mathematics that deals with derivatives and integrals of arbitrary orders. Fractional differential equations have been of great interest because of their intensive development of fractional calculus and its applications [1-11].

Recently, the quadratic perturbation of nonlinear differential equations (called hybrid differential equations) has captured much attention. The importance of the investigations of hybrid differential equation lies in the fact that they include several dynamic systems as special cases. Dhage and Lakshmikantham [12] discussed the existence and uniqueness theorems of the solution to the ordinary first-order hybrid differential equation with perturbation of first type

$$
\frac{d}{d t}\left[\frac{x(t)}{f(t, x(t))}\right]=g(t, x(t)), \quad \text { a.e. } t \in J, x\left(t_{0}\right)=x_{0} \in \mathbb{R}
$$

where $f \in C(J \times \mathbb{R}, \mathbb{R}-\{0\})$ and $g \in \mathscr{C}(J \times \mathbb{R}, \mathbb{R})$, where $J=\left[t_{0}, t_{0}+a\right]$ is a bounded interval in $\mathbb{R}$ for some $t_{0}$ and $a \in \mathbb{R}$ with $a>0, C(J \times \mathbb{R}, \mathbb{R})$ is the class of continuous functions and $\mathscr{C}(J \times \mathbb{R}, \mathbb{R})$ is called the Caratheodory class of functions $g: J \times \mathbb{R} \rightarrow \mathbb{R}$ which are Lebesgue integrable bounded by a Lebesgue integrable function on J. Moreover

(i) the map $t \mapsto g(t, x)$ is measurable for each $x \in \mathbb{R}$;

(ii) the map $x \mapsto g(t, x)$ is continuous for each $t \in J$.

Dhage and Jadhav [13] discussed the existence and uniqueness theorems of the solution of the ordinary first-order hybrid differential equation with perturbation of second type

$$
\begin{aligned}
\frac{d}{d t}[x(t)-f(t, x(t))] & =g(t, x(t)), \quad \text { a.e. } t \in J, x\left(t_{0}\right) \\
& =x_{0} \in \mathbb{R}
\end{aligned}
$$

Fractional hybrid differential equations have been studied using Riemman-Liouville derivative in literature (see, e.g., [14-16] and the references therein). Ammi et al. [14] discussed 
a generalization of (1) by replacing the ordinary by fractional derivative in Riemann-Liouvile sense with delay in the form

$$
\begin{gathered}
\frac{d^{\alpha}}{d t^{\alpha}}\left[\frac{x(t)}{f(t, x(t))}\right]=g\left(t, x_{t}, \int_{0}^{t} k\left(s, x_{s}\right) d s\right) \\
\text { a.e., } t \in I=[0, T],
\end{gathered}
$$

subject to $x(t)=\phi(t), \quad t \in I_{0}=[-\delta, 0]$,

where $\phi \in C\left(I_{0}\right)$ and $x_{t}: I_{0} \rightarrow \mathbb{C}$ is the continuous function defined by $x_{t}(\theta)=x(t+\theta)$ for all $\theta \in I_{0}$. They used that $x(t)$ is a solution to (3) if and only if it is a solution to the integral equation

$$
x(t)=f(t, x(t)) I^{\alpha}\left(g\left(t, x_{t}, \int_{0}^{t} k\left(s, x_{s}\right) d s\right)\right) ;
$$

then they studied this integral equation. However, it is easy to see that this concept of a solution is not realistic because of neglecting the initial condition. In fact, if we consider the following trivial example with $f(t, x(t))=$ $1, g\left(t, x_{t}, \int_{0}^{t} k\left(s, x_{s}\right) d s\right)=0$ we get the function $x(t)=$ $\left.\left(I^{1-\alpha} x(t)\right)\right|_{t=0}\left(t^{\alpha-1} / \Gamma(\alpha)\right)$ which is a solution to (3) but not to (4).

Lu et al. [15] discussed a generalization of (2) by replacing the classical differentiation by fractional derivative in the Riemann-Liouvile sense as

$$
\frac{d^{\alpha}}{d t^{\alpha}}[x(t)-f(t, x(t))]=g(t, x(t)), \quad t \in J=\left[t_{0}, t_{0}+a\right],
$$

subject to $x\left(t_{0}\right)=x_{0}$.

They proved that $x(t)$ is a solution to (5) if and only if it is a solution to the integral equation

$$
\begin{aligned}
x(t)= & x_{0}-f\left(t_{0}, x_{0}\right)+f(t, x(t)) \\
& +\frac{1}{\Gamma(\alpha)} \int_{t_{0}}^{t}(t-s)^{\alpha-1} g(s, x(s)) d s, \quad t \in J ;
\end{aligned}
$$

then they studied this integral equation. Again, we recall that this concept of a solution is not realistic because of neglecting the initial condition. In fact, consider the following trivial example with $f(t, x(t))=1, g(t, x(t))=0, t_{0}=0$; we get that the function $x(t)=1+\left.\left(I^{1-\alpha}(x(t)-1)\right)\right|_{t=0}\left(t^{\alpha-1} / \Gamma(\alpha)\right)$ is a solution to (5) but not for (6).

Here we discuss the existence of solutions to hybrid fractional differential equations in both types using the Caputo fractional derivative instead of the classical one in both (1) and (2).

Our paper is organized as follows. In Section 2 some basic definitions and theorems are given. Fractional hybrid differential equation of type 1 is discussed in Section 3 while in Section 4 we discuss the fractional hybrid differential equation of type 2. Our conclusion is presented in Section 5.

\section{Preliminaries}

Below we present some definitions and theorems used in the rest of this paper.
Definition 1 (see $[1,8-11]$ ). If $f(t) \in L^{1}(a, b)$, the set of all integrable functions, and $\alpha>0$ then the left RiemannLiouville fractional integral of order $\alpha$, is defined by

$$
I^{\alpha} f(t)=\frac{1}{\Gamma(\alpha)} \int_{a}^{t}(t-\tau)^{\alpha-1} f(\tau) d \tau .
$$

Definition 2 (see [8-11]). For $\alpha>0$ the left Riemann-Liouville fractional derivative of order $\alpha$ is defined by

$$
\frac{d^{\alpha}}{d t^{\alpha}} f(t)=\frac{1}{\Gamma(n-\alpha)} D^{n} \int_{a}^{t}(t-\tau)^{n-\alpha-1} f(\tau) d \tau,
$$

where $n$ is such that $n-1<\alpha<n$ and $D=d / d t$.

Definition 3 (see [8-11]). For $\alpha>0$ the left Caputo fractional derivative of order $\alpha$ is defined by

$$
D^{\alpha} f(t)=\frac{1}{\Gamma(n-\alpha)} \int_{a}^{t}(t-\tau)^{n-\alpha-1} D^{n} f(\tau) d \tau,
$$

where $n$ is such that $n-1<\alpha<n$ and $D=d / d \tau$.

The following two fixed point theorems are used in our paper (see $[12,13,17-19])$.

Theorem 4. Let $\mathscr{B}_{r}(0)$ and $\overline{\mathscr{B}_{r}(0)}$ be open and closed balls in a Banach algebra $X$ centered at origin 0 of radius $r$, for some real number $r>0$, and let $A, B: \overline{\mathscr{B}_{r}(0)} \rightarrow X$ be two operators satisfying the following.

(a) A is Lipschitz with Lipschitz constant $\gamma$;

(b) $B$ is continuous and compact;

(c) $\gamma M<1$, where $M=\left\|B\left(\overline{\mathscr{B}_{r}(0)}\right)\right\|=\sup \{\|B(x)\|: x \in$ $\left.\overline{\mathscr{B}_{r}(0)}\right\}$.

Then, either

(i) the equation $A x B x=x$ has a solution in $\overline{\mathscr{B}_{r}(0)}$, or

(ii) there is an element $x \in X$ such that $\|x\|=r$ satisfying $\lambda A x B x=x$, for some $0<\lambda<1$.

Definition 5. Let $X$ be a Banach space. A mapping $T: X \rightarrow$ $X$ is called $\mathscr{D}$-Lipschitzian if there exists a continuous and nondecreasing function $\phi: \mathbb{R}^{+} \rightarrow \mathbb{R}^{+}$such that $\| T x-$ $T y \| \leq \phi(\|x-y\|)$ for all $x, y \in X$, where $\phi(0)=0$. If $\phi$ is not necessarily nondecreasing and satisfies $\phi(r)<r$, for $r>0$, the mapping $T$ is called a nonlinear contraction with a contraction function $\phi$.

Theorem 6. Let $S$ be a closed convex and bounded subset of the Banach space $X$ and let $A: X \rightarrow X$ and $B: S \rightarrow X$ be two operators such that

(a) A is nonlinear contraction,

(b) $B$ is continuous and compact, and

(c) $x=A x+B y$ for all $y \in S \Rightarrow x \in S$.

Then the operator equation $A x+B x=x$ has a solution in $S$. 


\section{Fractional Hybrid Differential Equation of the First Type}

Consider the fractional hybrid differential equation in the form

$$
\begin{array}{r}
D^{\alpha}\left[\frac{x(t)}{f(t, x(t))}\right]=h(t), \quad \text { a.e. } t \in J=[0, T], \\
x(0)=x_{0} \in \mathbb{R} ;
\end{array}
$$

we have the following Lemma.

Lemma 7. Any function satisfies (10) with $h \in L^{1}\left(J, \mathbb{R}^{+}\right)$will also satisfy the integral equation

$$
\begin{aligned}
& x(t) \\
& =f(t, x(t))\left(\frac{x_{0}}{f\left(0, x_{0}\right)}+\int_{0}^{t} \frac{(t-s)^{\alpha-1}}{\Gamma(\alpha)} h(s) d s\right), \quad t \in J .
\end{aligned}
$$

In addition if the function $x \mapsto x / f(0, x)$ is injective, and $I^{\alpha} h(t)$ is an absolutely continuous function, then the converse is true.

Proof. Assume that $x(t)$ satisfies (10). Then, $(x(t) / f(t, x(t)))$ is absolutely continuous that we get that $D^{\alpha}(x(t) / f(t, x(t)))$ exists and is Lebesgue integrable on $J$. Applying the fractional integration $I^{\alpha}$ to both sides of (10) we get (11).

Conversely, assume that $x(t)$ satisfies (11) with $I^{\alpha} h(t)$ is absolutely continuous we get that $(x(t) / f(t, x(t)))=$ $\left(x_{0} / f\left(0, x_{0}\right)\right)+\int_{0}^{t}\left((t-s)^{\alpha-1} / \Gamma(\alpha)\right) h(s) d s$ is absolutely continuous. Then by differentiating both sides and then operating by the fractional integration $I^{1-\alpha}$ we get

$$
D^{\alpha}\left[\frac{x(t)}{f(t, x(t))}\right]=h(t),
$$

and for the initial condition substitute by $t=0$ in (11) we get

$$
\frac{x(0)}{f(0, x(0))}=\frac{x_{0}}{f\left(0, x_{0}\right)},
$$

and since $x \mapsto(x / f(0, x))$ is injective then $x(0)=x_{0}$.

Now consider the fractional hybrid differential equation of first type in the form

$$
\begin{array}{r}
D^{\alpha}\left[\frac{x(t)}{f(t, x(t))}\right]=g(t, x(t)), \quad \text { a.e. } t \in J=[0, T], \\
x(0)=x_{0} \in \mathbb{R},
\end{array}
$$

where, $f \in C(J \times \mathbb{R}, \mathbb{R}-0)$ and $g \in \mathscr{C}(J \times \mathbb{R}, \mathbb{R})$.

Definition 8. (1) The function $x \in C(J, \mathbb{R})$ is called a mild solution of the hybrid differential equation of first type (14) if it satisfies the integral equation

$$
x(t)=f(t, x(t))\left(\frac{x_{0}}{f\left(0, x_{0}\right)}+\int_{0}^{t} \frac{(t-s)^{\alpha-1}}{\Gamma(\alpha)} g(s, x(s)) d s\right) .
$$

(2) The function $x \in A C(J, \mathbb{R})$, the space of absolutely continuous real-valued functions defined on $J$, is called a strong solution of (14) if

(a) the function $t \mapsto(x / f(t, x))$ is absolutely continuous for each $x \in \mathbb{R}$, and

(b) $x$ satisfies (14).

Theorem 9. Assume the following.

(H1) There exists a constant $\gamma>0$ such that $\mid f(t, x)-$ $f(t, y)|\leq \gamma| x-y \mid$ for all $t \in J$ and $x, y \in \mathbb{R}$.

(H2) There exists a function $h \in L^{1}(J, \mathbb{R})$ such that $|g(t, x)| \leq h(t)$ a.e. $t \in J$ for all $x \in \mathbb{R}$.

(H3) $\gamma\left(\left|x_{0} / f\left(0, x_{0}\right)\right|+\left(T^{\alpha} / \Gamma(\alpha+1)\right)\|h\|_{L^{1}}\right)<1$.

(H4) There exists $r>0$ such that $r>\left(F_{0}\left(\left|x_{0} / f\left(0, x_{0}\right)\right|+\right.\right.$ $\left.\left.\left(T^{\alpha} / \Gamma(\alpha+1)\right)\|h\|_{L^{1}}\right)\right) /\left(1-\gamma\left(\left|x_{0} / f\left(0, x_{0}\right)\right|+\left(T^{\alpha} / \Gamma(\alpha+\right.\right.\right.$ 1)) $\left.\left.\|h\|_{L^{1}}\right)\right)$, where $F_{0}=\sup _{t \in J}|f(t, 0)|$.

Then (14) has a mild solution on J.

Proof. Let $\mathscr{B}_{r}(0)$ be an open ball centered at the origin and of radius $r>0$ in the Banach algebra $X=C(J, \mathbb{R})$ (the Banach space of continuous valued functions defined on the interval $J$ equipped with the sup-norm, $\|x\|=\sup _{s \in J}|x(s)|$, and with multiplication defined by $(x y)(s)=x(s) y(s)$, for $s \in J)$. We prove the existence of a mild solution to problem (14) by discussing the solution to the integral equation (15) which is equivalent to the operator equation

$$
A x(t) B x(t)=x(t), \quad t \in J,
$$

where $A, B: \overline{\mathscr{B}_{r}(0)} \rightarrow X$ are defined by

$$
\begin{gathered}
A x(t)=f(t, x(t)), \\
B x(t)=\frac{x_{0}}{f\left(0, x_{0}\right)}+\int_{0}^{t} \frac{(t-s)^{\alpha-1}}{\Gamma(\alpha)} g(s, x(s)) d s .
\end{gathered}
$$

Now we divide our proof in several steps.

Step 1. The operator $A$ is Lipschitz on $X$.

Let $x, y \in X$ and $t \in J$; then by (H1) we get

$$
\begin{aligned}
|A x(t)-A y(t)| & =|f(t, x(t))-f(t, y(t))| \\
& \leq \gamma|x(t)-y(t)| \leq \gamma\|x-y\| .
\end{aligned}
$$

Taking the supremum over $t \in J$ we get that $A$ is Lipschitz on $X$ with Lipschitz constant $\gamma$.

Step 2. The operator $B$ is continuous operator on $\overline{\mathscr{B}_{r}(0)}$.

Let $\left\{x_{n}\right\}$ be a convergent sequence in $\overline{\mathscr{B}_{r}(0)}$ converging to $x \in \overline{\mathscr{B}_{r}(0)}$. Then, by the Lebesgue dominated converging theorem,

$$
\begin{aligned}
& \lim _{n \rightarrow \infty} B x_{n}(t) \\
& =\lim _{n \rightarrow \infty}\left(\frac{x_{0}}{f\left(0, x_{0}\right)}+\int_{0}^{t} \frac{(t-s)^{\alpha-1}}{\Gamma(\alpha)} g\left(s, x_{n}(s)\right) d s\right) \\
& =\frac{x_{0}}{f\left(0, x_{0}\right)}+\int_{0}^{t} \frac{(t-s)^{\alpha-1}}{\Gamma(\alpha)} \lim _{n \rightarrow \infty} g\left(s, x_{n}(s)\right) d s
\end{aligned}
$$




$$
\begin{aligned}
& =\frac{x_{0}}{f\left(0, x_{0}\right)}+\int_{0}^{t} \frac{(t-s)^{\alpha-1}}{\Gamma(\alpha)} g(s, x(s)) d s \\
& =B x(t),
\end{aligned}
$$

for all $t \in J$ with prove the continuity of the operator $B$.

Step 3. The operator $B$ is compact operator on $\overline{\mathscr{B}_{r}(0)}$.

Let $x$ be arbitrary in $\overline{\mathscr{B}_{r}(0)}$. By hypothesis $(\mathrm{H} 2)$ and using Young's inequality for convolutions we get

$$
\begin{aligned}
|B x(t)| & \leq\left|\frac{x_{0}}{f\left(0, x_{0}\right)}\right|+\int_{0}^{t} \frac{(t-s)^{\alpha-1}}{\Gamma(\alpha)}|g(s, x(s))| d s \\
& \leq\left|\frac{x_{0}}{f\left(0, x_{0}\right)}\right|+\int_{0}^{t} \frac{(t-s)^{\alpha-1}}{\Gamma(\alpha)} h(s) d s \\
& \leq\left|\frac{x_{0}}{f\left(0, x_{0}\right)}\right|+\frac{T^{\alpha}}{\Gamma(\alpha+1)}\|h\|_{L^{1}},
\end{aligned}
$$

which by taking the supremum over $t$ gives

$$
\|B x\| \leq\left|\frac{x_{0}}{f\left(0, x_{0}\right)}\right|+\frac{T^{\alpha}}{\Gamma(\alpha+1)}\|h\|_{L^{1}}, \quad \forall x \in \overline{\mathscr{B}_{r}(0)}
$$

which proves that $B\left(\overline{\mathscr{B}_{r}(0)}\right)$ is a uniformly bounded set in $X$. Now, we prove that $B\left(\overline{\mathscr{B}_{r}(0)}\right)$ is an equicontinuous set in $X$. For $0 \leq t_{1} \leq t_{2} \leq T$ we have

$$
\begin{aligned}
& \left|B x\left(t_{1}\right)-B x\left(t_{2}\right)\right| \\
& =\frac{1}{\Gamma(\alpha)} \mid \int_{0}^{t_{1}}\left(t_{1}-s\right)^{\alpha-1} g(s, x(s)) d s \\
& -\int_{0}^{t_{2}}\left(t_{2}-s\right)^{\alpha-1} g(s, x(s)) d s \\
& \leq \frac{1}{\Gamma(\alpha)} \mid \int_{0}^{t_{1}}\left(t_{1}-s\right)^{\alpha-1} g(s, x(s)) d s \\
& -\int_{0}^{t_{1}}\left(t_{2}-s\right)^{\alpha-1} g(s, x(s)) d s \\
& +\frac{1}{\Gamma(\alpha)} \mid \int_{0}^{t_{1}}\left(t_{2}-s\right)^{\alpha-1} g(s, x(s)) d s \\
& -\int_{0}^{t_{2}}\left(t_{2}-s\right)^{\alpha-1} g(s, x(s)) d s \\
& \leq \frac{\|h\|_{L^{1}}}{\Gamma\left(\alpha_{1}\right)}\left[\left|t_{2}^{\alpha}-t_{1}^{\alpha}-\left(t_{2}-t_{1}\right)^{\alpha}\right|+\left(t_{2}-t_{1}\right)^{\alpha}\right] .
\end{aligned}
$$

Hence, for $\epsilon>0$, there exists a $\delta>0$ such that

$$
\begin{array}{r}
\left|t_{1}-t_{2}\right|<\delta \Longrightarrow\left|B x\left(t_{1}\right)-B x\left(t_{2}\right)\right|<\epsilon, \\
\forall t_{1}, t_{2} \in J, \quad x \in \overline{\mathscr{B}_{r}(0)} .
\end{array}
$$

This shows that $B\left(\overline{\mathscr{B}_{r}(0)}\right)$ is an equicontinuous set in $X$. By the Arzela Ascoli Theorem we get that the operator $B$ is a compact operator.

Step 4. $\gamma M<1$, where $M=\left\|B\left(\overline{\mathscr{B}_{r}(0)}\right)\right\|=\sup \{\|B(x)\|: x \in$ $\left.\overline{\mathscr{B}_{r}(0)}\right\}$.

Using results in Step 3 we get

$$
\begin{aligned}
M=\left\|B\left(\overline{\mathscr{B}_{r}(0)}\right)\right\| & =\sup \left\{\|B(x)\|: x \in \overline{\mathscr{B}_{r}(0)}\right\} \\
& \leq\left|\frac{x_{0}}{f\left(0, x_{0}\right)}\right|+\frac{T^{\alpha}}{\Gamma(\alpha+1)}\|h\|_{L^{1}}
\end{aligned}
$$

which gives from the hypothesis (H3) that

$$
\gamma M \leq \gamma\left(\left|\frac{x_{0}}{f\left(0, x_{0}\right)}\right|+\frac{T^{\alpha}}{\Gamma(\alpha+1)}\|h\|_{L^{1}}\right)<1 .
$$

It remains to prove that the conclusion (ii) of Theorem 4 cannot be realizable.

Let $x \in X$ and $\lambda \in(0,1)$ be such that $\|x\|=r$ and $x=$ $\lambda A x B x$. It follows that

$|x(t)|$

$$
\begin{aligned}
= & \lambda\left|f(t, x(t))\left(\frac{x_{0}}{f\left(0, x_{0}\right)}+\int_{0}^{t} \frac{(t-s)^{\alpha-1}}{\Gamma(\alpha)} g(s, x(s)) d s\right)\right| \\
\leq & \lambda|f(t, x(t))|\left(\left|\frac{x_{0}}{f\left(0, x_{0}\right)}\right|+\left|\int_{0}^{t} \frac{(t-s)^{\alpha-1}}{\Gamma(\alpha)} g(s, x(s)) d s\right|\right) \\
\leq & \lambda|f(t, x(t))-f(t, 0)+f(t, 0)| \\
& \times\left(\left|\frac{x_{0}}{f\left(0, x_{0}\right)}\right|+\int_{0}^{t} \frac{(t-s)^{\alpha-1}}{\Gamma(\alpha)}|g(s, x(s))| d s\right) \\
\leq & \lambda\left(\gamma|x(t)|+F_{0}\right)\left(\left|\frac{x_{0}}{f\left(0, x_{0}\right)}\right|+\frac{T^{\alpha}}{\Gamma(\alpha+1)}\|h\|_{L^{1}}\right) \\
\leq & \frac{F_{0}\left(\left|x_{0} / f\left(0, x_{0}\right)\right|+\left(T^{\alpha} / \Gamma(\alpha+1)\right)\|h\|_{L^{1}}\right)}{1-\lambda \gamma\left(\left|x_{0} / f\left(0, x_{0}\right)\right|+\left(T^{\alpha} / \Gamma(\alpha+1)\right)\|h\|_{L^{1}}\right)} .
\end{aligned}
$$

Taking supremum over $t$ and using (H4) and $\lambda \in(0,1)$ we get

$$
\begin{aligned}
\|x\| & \leq \frac{F_{0}\left(\left|x_{0} / f\left(0, x_{0}\right)\right|+\left(T^{\alpha} / \Gamma(\alpha+1)\right)\|h\|_{L^{1}}\right)}{1-\lambda \gamma\left(\left|x_{0} / f\left(0, x_{0}\right)\right|+\left(T^{\alpha} / \Gamma(\alpha+1)\right)\|h\|_{L^{1}}\right)} \\
& <\frac{F_{0}\left(\left|x_{0} / f\left(0, x_{0}\right)\right|+\left(T^{\alpha} / \Gamma(\alpha+1)\right)\|h\|_{L^{1}}\right)}{1-\gamma\left(\left|x_{0} / f\left(0, x_{0}\right)\right|+\left(T^{\alpha} / \Gamma(\alpha+1)\right)\|h\|_{L^{1}}\right)}<r
\end{aligned}
$$

which contradicts $\|x\|=r$; thus (ii) of Theorem 4 is not possible; hence the operator $A x B x=x$ has a solution in $\overline{\mathscr{B}_{r}(0)}$. As a result problem (14) has a mild solution on $J$ which completes the proof of our theorem.

We finish this section by the following example. 
Example 10. Consider the fractional hybrid differential equation

$$
\begin{array}{r}
D^{0.5}\left(\frac{x(t)}{1+(\sin (t) / 16)|x(t)|}\right)=\frac{t x(t)}{1+|x(t)|}, \\
x(0)=1, \quad t \in J=[0, \pi] .
\end{array}
$$

It is easy to see that all hypotheses of Theorem 9 are satisfied with

$$
\gamma=\frac{1}{16}, \quad T=\pi, \quad h(t)=t, \quad F_{0}=1 .
$$

We conclude that

$$
\begin{aligned}
& \frac{F_{0}\left(\left|x_{0} / f\left(0, x_{0}\right)\right|+\left(T^{\alpha} / \Gamma(\alpha+1)\right)\|h\|_{L^{1}}\right)}{1-\gamma\left(\left|x_{0} / f\left(0, x_{0}\right)\right|+\left(T^{\alpha} / \Gamma(\alpha+1)\right)\|h\|_{L^{1}}\right)} \\
& =\frac{1+\pi^{2}}{1-(1 / 16)\left(1+\pi^{2}\right)}<34,
\end{aligned}
$$

hence (28) has a mild solution in $\overline{\mathscr{B}_{34}(0)}$.

\section{Fractional Hybrid Differential Equation of Second Type}

Consider the fractional hybrid differential equation in the form

$$
\begin{array}{r}
D^{\alpha}[x(t)-f(t, x(t))]=h(t), \quad \text { a.e. } t \in J=[0, T], \\
x(0)=x_{0} \in \mathbb{R} ;
\end{array}
$$

similar to Lemma 7 we can prove

Lemma 11. Any function satisfies (31) with $h \in L^{1}\left(J, \mathbb{R}^{+}\right)$will also satisfy the integral equation

$$
\begin{aligned}
x(t)= & f(t, x(t))+x_{0}-f\left(0, x_{0}\right) \\
& +\int_{0}^{t} \frac{(t-s)^{\alpha-1}}{\Gamma(\alpha)} h(s) d s, \quad t \in J .
\end{aligned}
$$

In addition if the function $x \mapsto x-f(0, x)$ is injective, and $I^{\alpha} h(t)$ is an absolutely continuous function then the converse is true.

Now consider the fractional hybrid differential equation of second type in the form

$$
\begin{array}{r}
D^{\alpha}[x(t)-f(t, x(t))]=g(t, x(t)), \quad \text { a.e. } t \in J=[0, T], \\
x(0)=x_{0} \in \mathbb{R},
\end{array}
$$

where, $f \in C(J \times \mathbb{R}, \mathbb{R}-0)$ and $g \in \mathscr{C}(J \times \mathbb{R}, \mathbb{R})$.

Definition 12. One has the following functions.

(1) The function $x \in C(J, \mathbb{R})$ is called a mild solution of the hybrid differential equation of second type (33) if it satisfies the integral equation

$$
\begin{aligned}
x(t)= & f(t, x(t))+x_{0}-f\left(0, x_{0}\right) \\
& +\int_{0}^{t} \frac{(t-s)^{\alpha-1}}{\Gamma(\alpha)} g(s, x(s)) d s .
\end{aligned}
$$

(2) The function $x \in A C(J, \mathbb{R})$ is called a strong solution of (14) if

(a) the function $t \mapsto x-f(t, x)$ is absolutely continuous for each $x \in \mathbb{R}$, and

(b) $x$ satisfies (33).

Theorem 13. Assume the following.

(A1) There exists constants $M \geq L>0$ such that $\mid f(t, x)-$ $f(t, y) \mid \leq(L|x-y| /(M+|x-y|))$ for all $t \in J$ and $x, y \in \mathbb{R}$.

(A2) There exists a function $h \in L^{1}(J, \mathbb{R})$ such that $|g(t, x)| \leq h(t)$ a.e. $t \in J$ for all $x \in \mathbb{R}$.

(A3) There exists $r>0$ such that $r>\left|x_{0}-f\left(0, x_{0}\right)\right|+L+$ $F_{0}+\left(T^{\alpha} / \Gamma(\alpha+1)\right)\|h\|_{L^{1}}$ where $F_{0}=\sup _{t \in J}|f(t, 0)|$.

Then (33) has a mild solution on J.

Proof. Set $X=C(J, \mathbb{R})$ and define the set $S \subset X$ by $S=\{x \in$ $X:\|x\| \leq r\}$. We prove the existence of a mild solution to problem (33) by discussing the solution to the integral equation (34) which is equivalent to the operator equation

$$
A x(t)+B x(t)=x(t), \quad t \in J,
$$

where

$$
\begin{gathered}
A x(t)=f(t, x(t)), \\
B x(t)=x_{0}-f\left(0, x_{0}\right)+\int_{0}^{t} \frac{(t-s)^{\alpha-1}}{\Gamma(\alpha)} g(s, x(s)) d s .
\end{gathered}
$$

Now we prove our theorem by proving that the conditions of Theorem 6 are satisfied.

(a) Using the hypothesis (A1) we get

$$
\begin{aligned}
|A x(t)-A y(t)| & =|f(t, x(t))-f(t, y(t))| \\
& \leq \frac{L|x(t)-y(t)|}{M+|x(t)-y(t)|} \leq \frac{L\|x-y\|}{M+\|x-y\|},
\end{aligned}
$$

thus the operator $A$ is a nonlinear contraction with the function $\phi$ defined by $\phi(r)=\operatorname{Lr} /(M+r)$.

(b) Similarly, in proving Theorem 9 we can prove that $B$ is continuous and compact. 
(c) Let $x \in X$ be fixed and $y \in S$ be arbitrary such that $x=A x+B y$. Then we get

$|x(t)|$

$$
\begin{aligned}
& \leq|A x(t)|+|B y(t)| \leq|f(t, x(t))|+\left|x_{0}-f\left(0, x_{0}\right)\right| \\
& \quad+\int_{0}^{t} \frac{(t-s)^{\alpha-1}}{\Gamma(\alpha)}|g(s, y(s))| d s \\
& \leq|f(t, x(t))-f(t, 0)|+|f(t, 0)|+\left|x_{0}-f\left(0, x_{0}\right)\right| \\
& \quad+\int_{0}^{t} \frac{(t-s)^{\alpha-1}}{\Gamma(\alpha)} h(s) d s \\
& \leq L+F_{0}+\left|x_{0}-f\left(0, x_{0}\right)\right|+\frac{T^{\alpha}}{\Gamma(\alpha+1)}\|h\|_{L^{1}} \\
& \leq r
\end{aligned}
$$

which proves that $\|x\| \leq r$; thus $x \in S$.

Thus the conditions of Theorem 6 are satisfied; then the operator equation $A x(t)+B x(t)=x(t)$ has a solution in $S$ which proves the existence of a mild solution to problem (33) in $J$.

We finish this section with the following example.

Example 14. Consider the fractional hybrid differential equation

$$
\begin{array}{r}
D^{0.5}\left(x(t)-\frac{\sin (t)|x(t)|}{2+|x(t)|}\right)=\frac{t x(t)}{1+|x(t)|}, \\
x(0)=1, \quad t \in J=[0, \pi] ;
\end{array}
$$

we get that

$$
\begin{aligned}
& |f(t, x(t))-f(t, y(t))| \\
& \leq \frac{|x(t)|}{2+|x(t)|}-\frac{|y(t)|}{2+|y(t)|} \\
& \leq \frac{|x(t)-y(t)|+|y(t)|}{2+|x(t)-y(t)|+|y(t)|}-\frac{|y(t)|}{2+|x(t)-y(t)|+|y(t)|} \\
& \leq \frac{|x(t)-y(t)|}{2+|x(t)-y(t)|+|y(t)|} \\
& \leq \frac{|x(t)-y(t)|}{2+|x(t)-y(t)|}
\end{aligned}
$$

and where $|g(t, x(t))| \leq t$ we get that all hypotheses of Theorem 13 are satisfied with

$$
L=1, \quad M=2, \quad T=\pi, \quad h(t)=t, \quad F_{0}=0 .
$$

We conclude that

$$
\left|x_{0}-f\left(0, x_{0}\right)\right|+L+F_{0}+\frac{T^{\alpha}}{\Gamma(\alpha+1)}\|h\|_{L^{1}}=2+\pi^{2}<12 ;
$$

hence (39) has a mild solution in $S=\{x \in X:\|x\| \leq 12\}$.

\section{Conclusions}

In this paper we gave definitions of both strong and mild solutions to the fractional hybrid boundary value problems in two types using the Caputo fractional derivative of order $\alpha \in(0,1)$ and then we discussed the existence of at least one mild solution for each type. We gave examples proving the importance of taking into account of the initial conditions, therefore our results are realistic. We mention that in $[14,15]$ the initial conditions were neglected.

\section{Conflict of Interests}

The authors declare that there is no conflict of interests regarding the publication of this paper.

\section{References}

[1] D. Baleanu, K. Diethelm, E. Scalas, and J. J. Trujillo, Fractional Calculus Models and Numerical Methods, Series on Complexity, Nonlinearity and Chaos, World Scientific, 2012.

[2] C. Cattani, "Fractional calculus and Shannon wavelet," Mathematical Problems in Engineering, vol. 2012, Article ID 502812, 26 pages, 2012.

[3] M. A. E. Herzallah, A. M. A. El-Sayed, and D. Baleanu, "On the fractional-order diffusion-wave process," Romanian Journal in Physics, vol. 55, no. 3-4, pp. 274-284, 2010.

[4] M. A. E. Herzallah and D. Baleanu, "Existence of a periodic mild solution for a nonlinear fractional differential equation," Computers and Mathematics with Applications, vol. 64, no. 10, pp. 3059-3064, 2012.

[5] M. A. E. Herzallah, M. El-Shahed, and D. Baleanu, "Mild and strong solutions for a fractional nonlinear Neumann boundary value problem," Journal of Computational Analysis and Applications, vol. 15, no. 2, pp. 341-352, 2013.

[6] M. A. E. Herzallah, "Mild and strong solutions to few types of fractional order nonlinear equations with periodic boundary conditions," Indian Journal of Pure and Applied Mathematics, vol. 43, no. 6, pp. 619-635, 2012.

[7] R. Hilfer, Applications of Fractional Calculus in Physics, World Scientific, New Jersey, NJ, USA, 2000.

[8] A. A. Kilbas, H. M. Srivastava, and J. J. Trujillo, Theory and Applications of Fractional Differential Equations, vol. 204 of North-Holland Mathematical Studies, Elsevier, Amsterdam, The Netherlands, 2006.

[9] K. S. Miller and B. Ross, An Introduction to the Fractional Calculus and Fractional Differential Equations, John Wiley and Sons, New York, NY, USA, 1993.

[10] I. Podlubny, Fractional Differential Equations, Academic Press, New York, NY, USA, 1999.

[11] S. G. Samko, A. A. Kilbas, and O. I. Marichev, Fractional Integrals and Derivatives: Theory and Applcations, Gordon and Breach, New York, NY, USA, 1993.

[12] B. C. Dhage and V. Lakshmikantham, "Basic results on hybrid differential equations," Nonlinear Analysis: Hybrid Systems, vol. 4, no. 3, pp. 414-424, 2010.

[13] B. Dhage and N. Jadhav, "Basic results in the theory of hybrid differential equations with linear perturbations of second type," Tamkang Journal of Mathematics, vol. 44, no. 2, pp. 171-186, 2013. 
[14] M. R. Ammi, E. El Kinani, and D. F. Torres, "Existence and uniqueness of solutions to functional integro-differential fractional equations," Electronic Journal of Differential Equations, vol. 2012, no. 103, pp. 1-9, 2012.

[15] H. Lu, S. Sun, D. Yang, and H. Teng, "Theory of fractional hybrid differential equations with linear perturbations of second type," Boundary Value Problems, vol. 2013, article 23, 2013.

[16] Y. Zhao, S. Sun, Z. Han, and Q. Li, "Theory of fractional hybrid differential equations," Computers and Mathematics with Applications, vol. 62, no. 3, pp. 1312-1324, 2011.

[17] B. C. Dhage, "A fixed point theorem in Banach algebras involving three operators with applications," Kyungpook Mathematical Journal, vol. 44, no. 1, pp. 145-155, 2004.

[18] B. C. Dhage, "On a fixed point theorem in Banach algebras with applications," Applied Mathematics Letters, vol. 18, no. 3, pp. 273-280, 2005.

[19] B. C. Dhage, "Nonlinear functional boundary value problems in Banach algebras involving Caratheodories," Kyungpook Mathematical Journal, vol. 46, no. 4, pp. 527-541, 2006. 




Advances in

Operations Research

mansans

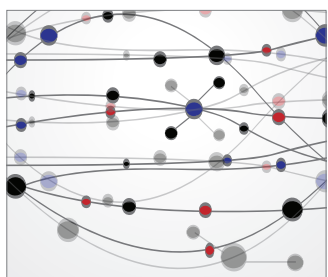

The Scientific World Journal
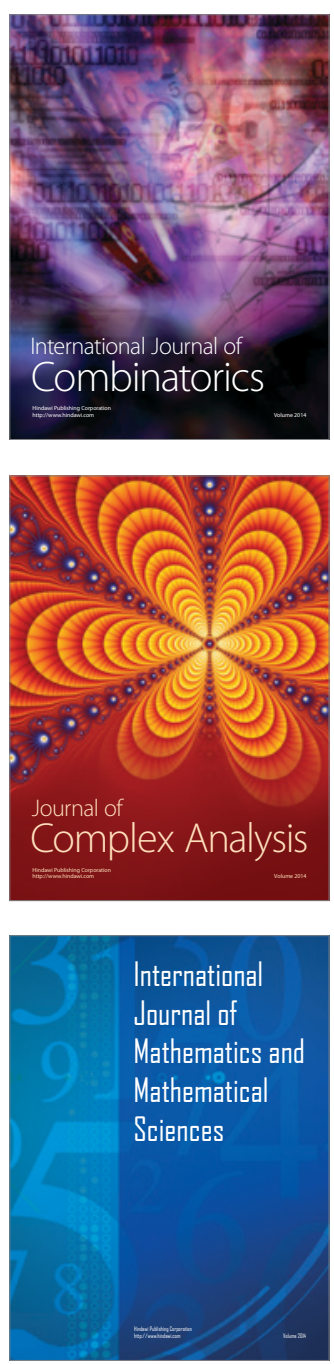
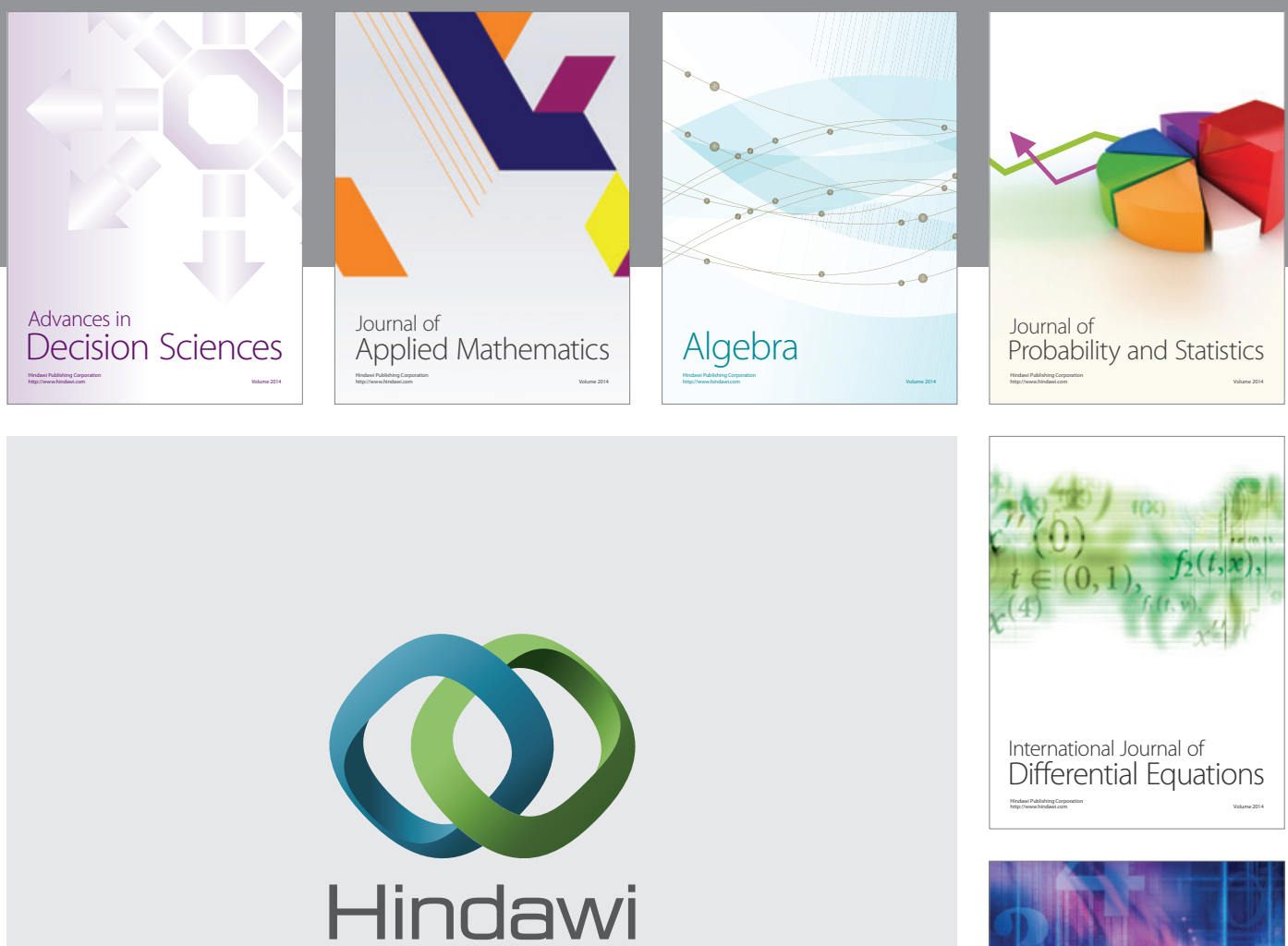

Submit your manuscripts at http://www.hindawi.com
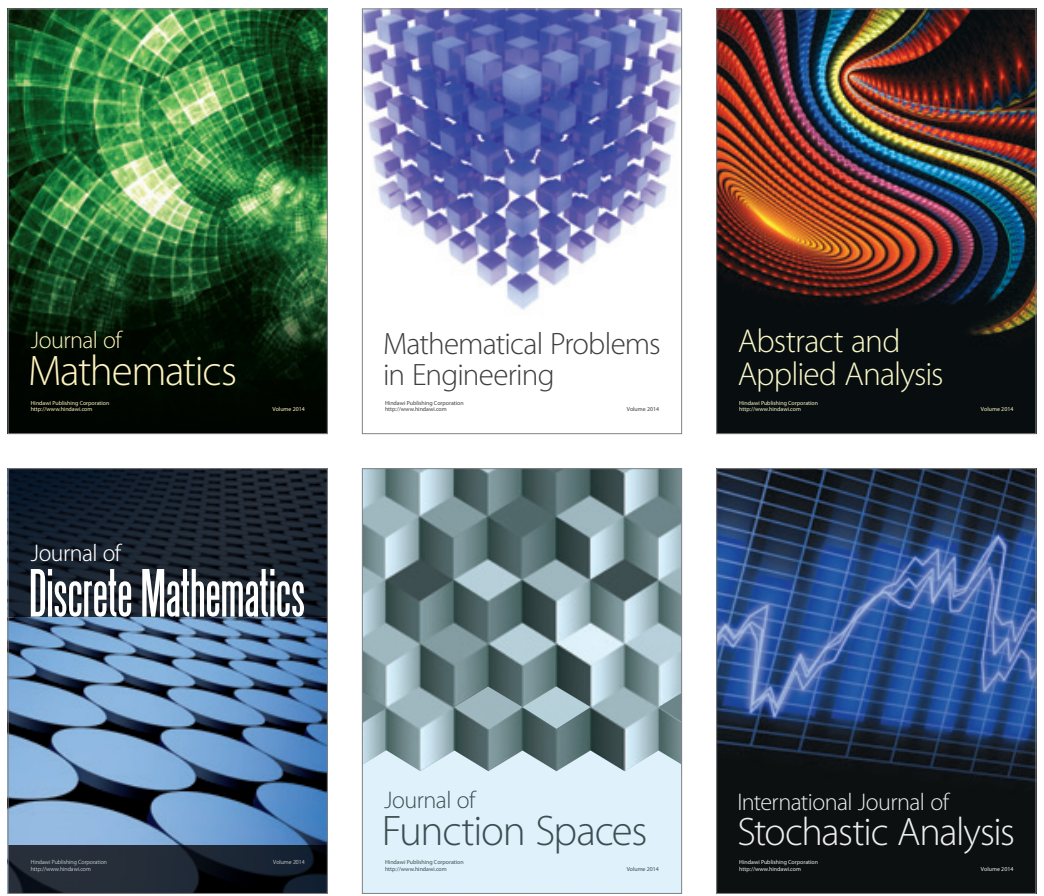

Journal of

Function Spaces

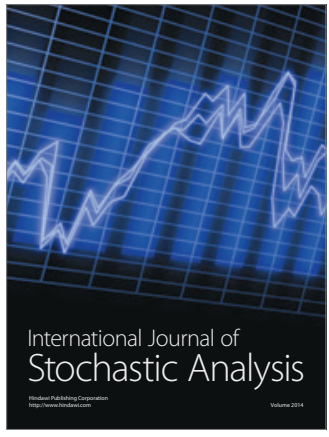

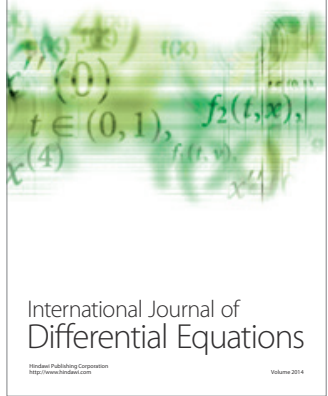
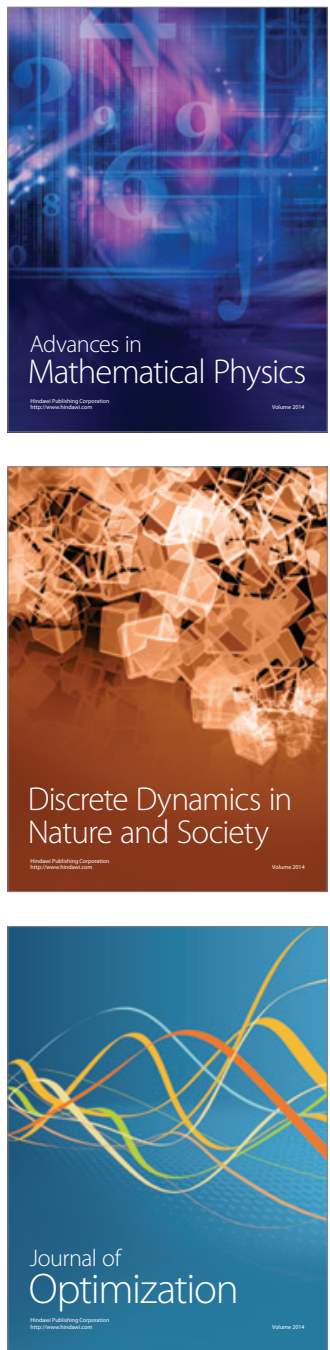